\title{
Circuit
}

Musiques contemporaines

\section{Musique actuelle et ambiguïté Musique actuelle and ambiguity}

\section{Dominique Olivier}

Volume 6, numéro 2, 1995

Musique actuelle?

URI : https://id.erudit.org/iderudit/902135ar

DOI : https://doi.org/10.7202/902135ar

Aller au sommaire du numéro

Éditeur(s)

Les Presses de l'Université de Montréal

ISSN

1183-1693 (imprimé)

1488-9692 (numérique)

Découvrir la revue

Citer cet article

Olivier, D. (1995). Musique actuelle et ambiguïté. Circuit, 6(2), 35-38.

https://doi.org/10.7202/902135ar

\section{Résumé de l'article}

Dans cette contribution résolument critique, l'auteure souligne le renoncement aux jugements de valeur qu'implique la pratique stylistique de la musique actuelle et dénonce l'ambiguïté créée par la conjonction de la dénonciation du commercial et l'utilisation des ressources de la musique industrielle. d'utilisation que vous pouvez consulter en ligne.

https://apropos.erudit.org/fr/usagers/politique-dutilisation/ 


\title{
PENSER LA MUSIQUE ACTUELLE ?
}

\section{Musique actuelle et ambiguïté}

\author{
Dominique Olivier
}

Il flotte sur la dénomination de "musique actuelle", et sur la notion qui la sous-tend, une ambiguilié encore bien loin d'être résolve. II n'est peut-être pas inutile de rappeler ici la liste des termes employés en histoire de la musique et qui soulignent une simultanéité entre notre existence propre et celle d'une forme d'expression artistique intrinsèquement liée à l'époque où nous vivons. Cohabitation, donc, entre l'art musical qui se falt et nous qui nous faisons en même temps, parallèlement. II y eut d'abord l'Ars nova, expression qui dit bien ce qu'elle veut dire en ce qu'elle désigne un art considéré à son époque comme révolutionnaire, ou comme "ayant un caractère de hardiesse propre à ce qui apparaît pour la première fois » (Dictionnaire Lexis). À cause toutefois du problème créé par cette désignation cinq siècles plus tard, à un âge où tout se voulait plus « nouveau » que par le passé, on a laissé l'appellation $d^{\prime}$ Ars nova à une partie bien circonscrite de la musique du XIV siècle, pour n'utiliser le terme d' "Art nouveau " que dans le domaine visuel. Au xx siècle où cette tendance à utiliser des termes exprimant la simultanéité est devenue généralisée, tout a commencé avec l'avènement de la « modernité » - l'adjectif moderne désignant une chose "qui appartient ou convient au temps présent ou à une époque récente, par opposition au passé ». On comprend la légitimité de la parole de Rimbaud : "Il faut être absolument moderne »-que le $x x^{e}$ siècle a faite sienne - et donc opposer une résistance à ce qui est ancien, sclérosé, inerte. Par contre, que peut devenir un art qui est qualifié de moderne, lorsqu'il appartient au passé ? Qu'à cela ne tienne, pour souligner son évolution, le vocabulaire musical en est venu à intégrer l'adjectif « contemporain » pour désigner une musique qui reniait totalement le passé et cherchait de nouvelles avenues créatrices - et même, sans vouloir accuser l'ironie de la chose, porteuses d'avenir. Mais si fréquemment les deux expressions peuvent désigner le même style musical, on situe les débuts de la modernité à une époque où la musique contemporaine, telle qu'on la conçoit aujourd'hui, n'existait pas encore. 
Devant l'échec du potentiel expressif de cette "modernité ", incluant bien sûr la musique dite contemporaine, une partie importante des compositeurs d'aujourd'hui en sont venus à retourner puiser derrière eux des matériaux culturels et sonores associés à des époques révolues, des éléments de langage appartenant au passé. De là est né le postmodernisme, dénomination qui traduit très bien l'impasse terminologique où nous nous trouvons. L'expression «musique d'aujourd'hui », qui n'est ni plus ni moins équivoque que les autres, n'est pas passée dans le vocabulaire musical autrement que comme synonyme permettant de varier un peu les formules employées, lorsqu'on parle de la musique contemporaine... - quand donc la musique " présente "? La musique "de maintenant »? La musique «immédiate »? etc.

La musique "actuelle », pour sa part, joue encore sur cette ambiguiité créée par sa définition. "Actuel : qui existe ou se produit dans le moment présent " (Dictionnaire Lexis). Cette ambiguîté naît de ce qu'on peut la comprendre de deux façons : soit dans la descendance des contemporains et des modernes - les musiciens de musique actuelle revendiquant un avant-gardisme qu'on ne reconnaît plus à la musique contemporaine, résolument versée du côté postmoderne ou devenue trop difficilement accessible -, ou dans le sens de musique qui n'existe que dans le moment présent, faisant allusion bien sûr au caractère improvisé des événements sonores qui la définissent. Les termes qui exprimaient une simultanéité, une proximité ou une nouveauté ont donc fini par créer une sorte de paradoxe terminologique. En voulant décrire une réalité présente, ces termes d'actualité devenaient la définition même de styles musicaux qui allaient inévitablement devenir dépassés, tôt ou tard. Nous sommes à une époque où les mots perdent peu à peu leur valeur sémantique pour devenir des étiquettes dépourvues de sens, ce que nous pouvons aisément associer à un des effets négatifs de la publicité /dans cet affaiblissement général du langage, les superlatifs font de plus en plus figure de normalité : tout n'est plus que super, extra ou ultra). Or, la musique actuelle est, plus que toutes les musiques dites d'avant-garde, un " art » victime du commerce, une forme de récupération insidieuse de ce qui aurait pu être dangereux en tant que forme de protestation sociale. Récupération insidieuse parce que se faisant souvent à l'insu même de ceux qui donnent vie à ce genre musical. En voulant dénoncer l' "engoncement » de la tradition et le compartimentage des styles au même titre que les intérêts commerciaux véhiculés par l'industrie de la musique rock, les instigateurs de cet éclectisme donnent dans le nivellement par le bas et l'abdication de tout jugement de valeur. Au diable la formation, le jugement, l'exigence intellectuelle ou même simplement l'effort. La musique actuelle ne cherche pas à exprimer des choses qui peuvent augmenter ou nourrir notre esprit critique : elle fait mine de dénoncer à la fois l'élitisme et la démagogie tout en jouant le jeu du commerce.

Nous ne voulons pas bien sûr remettre en cause l'honnêteté de beaucoup de ces acteurs du domaine de la musique actuelle, qui croient vraiment au 
rôle social qu'ils ont à jouer mais qui se leurrent malheureusement sur le sens de ce rôle. Ce n'est pas parce que la musique actuelle n'est pas immédiatement rentable pour beaucoup de ceux qui la font que l'on doit * automatiquement » la considérer comme marginale. La baisse de la qualité d'interprétation émanant souvent des événements de musique actuelle, par exemple, entraîne une baisse de l'exigence de l'auditeur et une perte d'acuité importante. Une fois cette acuité perdue, l'auditeur de musique actuelle en vient à ne plus différencier ce qui est bon de ce qui est mauvais et devient ainsi une proie de choix pour tous les courants musicaux moins nécessairement dénonciateurs... L'exemple parfait en fut le Festival Montréal Musiques Actuelles, qui a fait l'objet d'un numéro entier de la présente revue (vol. 1, $n^{\circ} 2,1990$ ). Dans ce déferlement de styles hétéroclites, dans cette tour de Babel où se côtoyaient les langages les plus différents, seul un auditeur averti et méfiant arrivait à séparer le bon grain de l'ivraie. Les autres, plus démunis, étaient livrés au seul critère du « j'aime ça, ou j'aime pas ça ». L'appellation de ce festival justifiait à elle seule que l'on y présente n'importe quoi, pourvu que l'on puisse y accoler l'étiquette $d^{\prime}$ ' actuel „ - un mot qui, comme nous avons tenté de le démontrer, porte facilement à confusion... Sous couvert d'ouverture d'esprit, que n'accepte-ton pas comme déchet culturel!

En vérité, comment la décrire, cette musique? Même en ayant fréquenté durant plusieurs années le Festival de musique actuelle de Victoriaville, l'exercice reste laborieux. Ce serait à la fois, dans beaucoup de cas, un ersatz de musique contemporaine et un ersatz de musique commerciale. Certaines visions empruntant plus à l'une qu'à l'autre, il serait tentant de les juger plutôt à l'aune du pourcentage d'éléments véritablement originaux qui s'y trouvent. Rarement, toutefois, peut-on y trouver autre chose qu'un remâchage d'ingrédients vieux comme l'histoire de la musique mais présentés sous l'aspect d'une bouillie informe dans laquelle il devient évidemment difficile de distinguer le caviar du reste d'omelette trop cuite.

Dans nombre d'événements de musique actuelle, on se trouve par conséquent en présence d'une forme d'égoïsme qui est le contraire de l'acte créateur : le compositeur, par métier, procède d'emblée à un énorme travail d'élagage, de sélection, ne livrant ultimement au monde que ce qu'il considère comme valable. L'improvisateur, lui, accapare son auditoire avec quantité de déchets sonores qui auraient été rejetés par le créateur avec la présomption d'arriver, peut-être, à quelques moments de grâce. Quand il ne recherche pas la grâce, l'improvisateur agit comme contestataire, rejetant les institutions et l'appareil commercial, sans proposer toutefois de solution de rechange. On jette tout à terre, mais sans songer à reconstruire. Ce rejet de la tradition, qui nous vient bien entendu essentiellement du modèle américain, nous rend immédiatement vulnérables aux intérêts commerciaux et au mauvais goût qui les accompagne souvent. 
La musique actuelle se nourrit donc d'une double ambiguité. La première émane de son appellation, qui nous fait hésiter entre musique improvisée qui existe au moment présent - et musique qui se fait aujourd'hui en exprimant les émotions véhiculées par le monde qui nous entoure, soit un art sonore plus actuel, plus près de nous que la musique moderne ou contemporaine. La seconde émane de son caractère à la fois dénonciateur et récupérateur. Étant d'une part un art qui " rue dans les brancards » et tente de faire réagir les auditeurs, d'autre part un univers sonore qui occulte l'exigence intellectuelle au profit d'une intégration acritique d'éléments de toutes provenances, la musique actuelle est à la fois « refus global » et « acceptation globale ». Mais laissons-lui tout de même ce qui lui revient: cette musique polymorphe, sans véritable existence propre et foncièrement adaptée au monde d'aujourd'hui, peutêtre est-elle véritablement ce qui exprime le mieux le malaise culturel au sein duquel nous sommes plongés. 